\title{
Evaluating the laparoscopic approach to proximally located benign gastric tumors
}

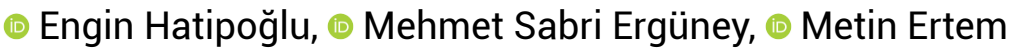 \\ Department of General Surgery, Istanbul University, Cerrahpaşa Faculty of Medicine, İstanbul, Turkey
}

\begin{abstract}
Introduction: The aim of this study was to evaluate the surgical and histopathological outcomes of proximally located benign gastric tumors.

Materials and Methods: This retrospective study included 14 patients with a proximally localized gastric tumor classified as Siewert III that was diagnosed between January 1, 2010 and December 31, 2019 in the general surgery clinics of Cerrahpasa Medicine Faculty Hospital or Acıbadem Kozyatağı Hospital. Patients who underwent laparoscopic and open surgery were included. Details of the patient characteristics, the clinical and pathological features of the tumors, and the patients' perioperative and postoperative results were recorded and analyzed.

Results: The most common cause of hospital admission was gastric bleeding (28.6\%). Localization of the tumor was the lesser curvature in $42.9 \%$, the posterior wall in $28.6 \%$, the anterior wall in $21.4 \%$, and the fundus in $7.1 \%$. Seven of the 10 laparoscopic operations were a hybrid-type procedure (endoscopic and laparoscopic). While 1 patient required admission to the intensive care unit, there was no mortality in the postoperative period. The pathological diagnosis was $64.3 \%(n=9)$ gastrointestinal stromal tumor, $14.2 \%$ $(n=2)$ schwannoma, $7.1 \%(n=1)$ leiomyoma, $7.1 \%(n=1)$ well-differentiated neuroendocrine tumor, and $7.1 \%$ $(n=1)$ enteric cyst. The surgical margin was clear in $92.9 \%(n=13)$ of the patients.

Conclusion: Resection of benign proximal gastric tumors was successful with laparoscopic endoscopic and open surgery. In this study, no major morbidity or mortality developed in the postoperative period. Although the superiority to open surgery has not yet been proven with long-term results, the laparoscopic endoscopic method should be the first choice due to known advantages, including ease of exploration.
\end{abstract}

Keywords: Benign gastric tumors; endoscopy; laparoscopy.

\section{Introduction}

Gastric cancers are common in the Asian population and also elderly people; however, despite the variations in incidence, early diagnosis and accurate treatment are critical. $^{[1-3]}$ Gastric tumors are classified with regard to their presence in the cardia (cardia and non-cardia). Cancers of the cardia are located in the proximal region of the gas- tric tract, and non-cardia cancers are located in the distal region of the stomach. ${ }^{[4]}$ Tumors with proximal localization are less common. Distally located tumors are thought to arise from chronic inflammation that usually develops on the background of chronic gastritis. The etiology of proximal cancers is still unclear. ${ }^{[5]}$

According to current data, gastric cancer is the third lead- 
ing cause of cancer-related death. ${ }^{[1]}$ The development of gastric cancer can be prevented and its progression may be slowed with life-style changes; thus, minimizing the risk of mortality in the event of early diagnosis and appropriate treatment. ${ }^{[4]}$ Today, the only curative option in the treatment of gastric tumors is surgery. Supporting gastric tumor surgery with chemotherapy and radiotherapy can increase the likelihood of successful treatment. ${ }^{[6]}$ As in many fields of surgery, minimally invasive methods have come to be preferred more frequently than classical open surgeries in benign gastric tumor resection. ${ }^{[7,8]}$ Different studies have shown that proximal gastric tumors have successful outcomes with laparoscopic approach. Compared to open surgical approaches, laparoscopic surgery has been reported to have many advantages, such as lower frequency of complications, shorter hospitalization, earlier return to daily life, and better aesthetic appearance. ${ }^{[7-10]}$

Gastric tumors with proximal localization are already rare; however, proximal tumors in which laparoscopic surgery can be used are even fewer. For this reason, it is very difficult to conduct large case series and analyses involving proximal gastric tumor cases resected by laparoscopic approach. The aim of this study was to evaluate the surgical outcomes and histopathological results of benign proximally located gastric tumors.

\section{Materials and Methods}

This study was carried out in 14 patients diagnosed between 1 January 2010 and 31 December 2019 in the general surgery clinics of Cerrahpasa Medicine Faculty Hospital and Acıbadem Kozyatağı Hospital with a proximally localized benign gastric tumor that found at Siewert 3 localization which is used in malign tumor classification. This is a descriptive, retrospective study based on medical records. Before the diagnosis, all patients underwent endoscopy. When necessary, the diagnoses were confirmed by using different methods including,endoscopic USG, magnetic resonance imaging (MRI), computed tomography (CT), positron emission tomography (PET) and biopsy. The definite diagnosis of "proximal gastric tumor" was based on the study by Siewert et al. and tumors within the lower 5 $\mathrm{cm}$ of the gastroesophageal line were considered as proximal gastric tumors. ${ }^{[1]}$

18 patients with benign gastric tumors were operated but 4 of them didn't come follow-ups and excluded from this study. Among the rest 14 proximal benign gastric tumors identified at our center. Patient characteristics, the clinical and pathological features of tumors, and perioperative and postoperative results were recorded. Informed consent was obtained from all individual participants included in the study. All procedures performed in studies were in accordance with the ethical standards of the institutional committee and with the 1964 Helsinki declaration and its later amendments or comparable ethical standards.

\section{Surgical Procedure}

The preferred surgical technique was determined according to the size and localization of each tumor. Before the operation, the localization of the tumor and whether it was located proximally was confirmed by gastrofiberoscope. In our study group, open or laparoscopic-endoscopic wedge resection approaches were preferred for tumor resection. The patients were placed in the supine position with legs spread the operating table and the operations were performed under general anesthesia. The surgeon was located between the legs of the patient or to the right of patient, changing places from time to time with the assistant holding the telescope. The endoscopist was positioned in the upper right of the patient, and the endoscopy and laparoscopy devices in the upper left. A total of 4 trocars were used: 10 $\mathrm{mm}$ for the camera, $10 \mathrm{~mm}$ for the liver retractor, and two of $5 \mathrm{~mm}$ for the working tools. Linear staplers were replaced from $5 \mathrm{~mm}$ trocar to $12 \mathrm{~mm}$ in the upper right quadrant during use. During the operation, the patient was given an inverted Trendelenburg position to the right. An ultrasonic scalpel was used to separate the gastrocolic ligament with the gastrosplenic ligament and divide the short gastric vessels. The amount of omentum dissected varied according to the localization of the tumor. In the posterior lesions, the liver retractor was placed to show the back side of the stomach and simultaneously eliminated the stomach and liver. Tumor borders, passage and stapler line were checked with the help of an endoscope. Linear staplers were used transversely to prevent narrowing of the stomach axis in lesser curvature and localizations close to the cardia. In some patients, $2^{\text {nd }}$ or $3^{\text {rd }}$ staplers were used depending on the size of the tumor. Nasogastric tube was placed in each patient. Stapler line was checked by giving methylene blue in patients those did not undergo to endoscopy. The drain was not used after the leak test in patients where we used single stapler. In patients where we used more than one stapler, a suture was placed in the stapler joints and a drain was placed. The tumor was taken out of the abdomen in the endobag via the $12 \mathrm{~mm}$ tracer site (Figs. 1-4). 


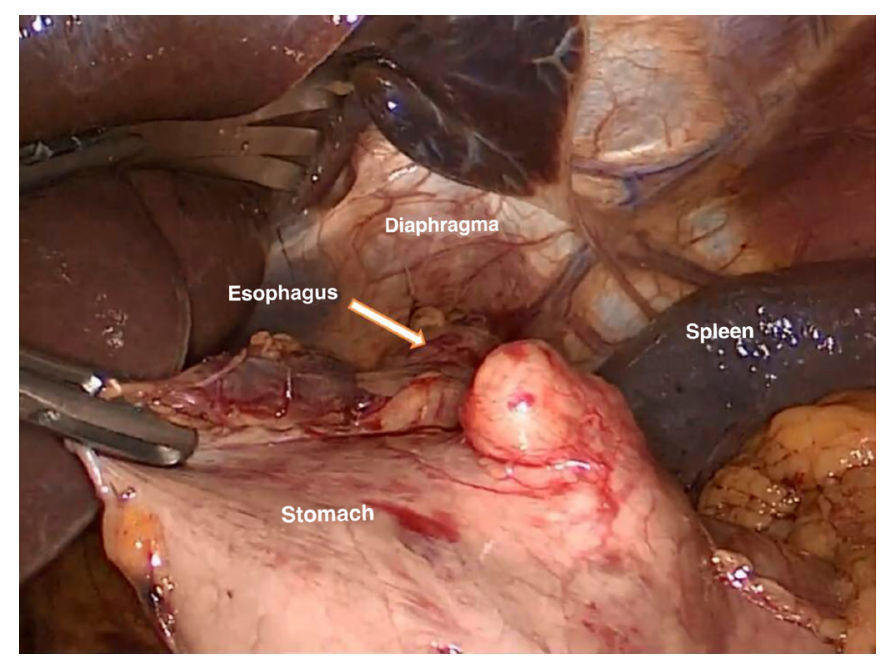

Figure 1. Determination of the fundus posterior wall located GIST after releasing the greater curvature.
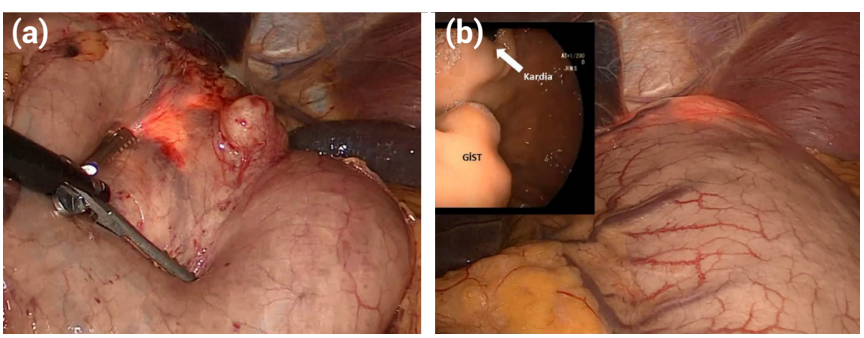

Figure 2 (a, b). Determination of clear surgical margin and passage transition of GIST from aerial plans with a laparoendoscopic method.
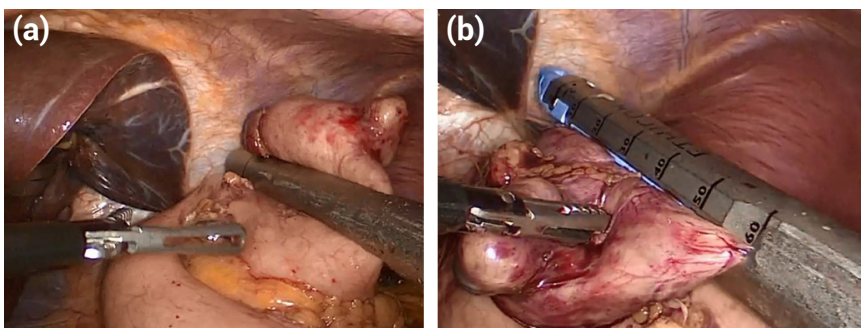

Figure 3 (a, b). Transection of GIST using linear stapler.

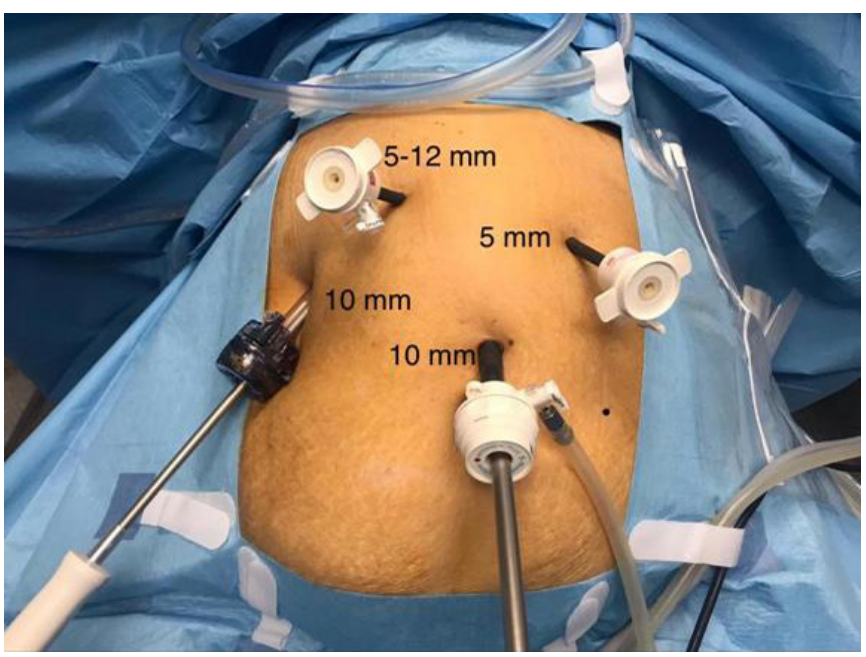

Figure 4. The locations of torakars used in operations.

\section{Postoperative Procedure}

After surgery, removal of the nasogastric tube and drain, the start of oral intake and patient discharge times were determined according to each patient's clinical characteristics. The pathological diagnosis of each patient was determined in the postoperative period by the same experienced pathologist in two centers. Form these evaluations, information such as immunohistochemical staining results (C-KIT, KI67, CD34, DOG1, SMA, Desmin, S100) tumor size, mitotic index, and condition of the surgical margin were recorded. A leakage was occurred at postop. stapler line in 1 case. After endoscopic stent placement and medical treatment management, this patient was discharged. One case was followed in the intensive care unit because of the postoperative comorbidities. Patients were called for follow-up at regular intervals and any complication, recurrence or mortality development was recorded due to the current operations.

\section{Statistical Analysis}

All analyses were performed on SPSS v21 (SPSS Inc., Chicago, IL, USA). Median, minimum and maximum values were used to describe continuous variables, and frequency and percentage values were used to show categorical variables.

\section{Results}

Among the 14 individuals, $42.9 \%(\mathrm{n}=6)$ were male, 57.1\% $(n=8)$ were women. Median age was 59 (39-72). The most common cause for hospital admission was gastric bleeding (28.6\%), followed by gastric complaints such as abdominal pain and dyspepsia in $21.4 \%(n=3)$ of the patients. Hypertension was diagnosed in $35.7 \%(n=5)$ of the patients. It was noted that $7.1 \%(n=1)$ had rheumatoid arthritis, 7.1\% (n=1) had toxic multinodular goiter and 7.1\% $(\mathrm{n}=1)$ had severe kyphosis-scoliosis. In regard to surgical histories, $14.2 \%$ of the participants $(n=2)$ had bypass, $14.2 \%(\mathrm{n}=2)$ had total abdominal hysterectomy with bilateral salpingo-oophorectomy, $7.1 \%(\mathrm{n}=1)$ had nephrectomy and $7.1 \%(n=1)$ had tonsillectomy. For diagnosis, all patients underwent endoscopy, 78.6\% ( $n=11)$ underwent endoscopic USG, 64.2\% ( $n=9)$ had CT imaging, 35.7\% $(n=5)$ underwent USG, 28.6\% ( $\mathrm{n}=4)$ had MR imaging and 21.4\% $(\mathrm{n}=3)$ underwent PET.

Localizations were as follows: $42.9 \%(\mathrm{n}=6)$ in the lesser curvature, $28.6 \%(n=4)$ on the posterior wall, $21.4 \%(n=3)$ on the anterior wall and $7.1 \%(n=1)$ in the fundus. Patients 
at risk (with regard to tumor localization and surgery) were operated on 2 to 26 months following diagnosis. None of the patients had postoperative bleeding. We checked the first three cases by giving the methylene blue to the stapler line. Next 7 of 10 laparoscopic operations were hybrid type (endoscopic and laparoscopic) There was no mortality in the postoperative period. During long-term followup, one patient developed peritoneal recurrence (Table 1).

The median size of the tumors removed was $4.0 \mathrm{~cm}$ (2.05.5). Pathological diagnoses were: $64.3 \%(n=9)$ gastrointestinal stromal tumor (GIST), 14.2\% ( $\mathrm{n}=2)$ schwannoma, $7.1 \%(\mathrm{n}=1)$ leiomyoma, 7.1\% ( $\mathrm{n}=1)$ enteric cyst, and 7.1\% $(\mathrm{n}=1)$ well-differentiated neuroendocrine tumor. One pa-

\section{Table 1. Demographic and clinical characteristics} of patients

\begin{tabular}{|c|c|}
\hline Age (years) & $59(39-72)$ \\
\hline \multicolumn{2}{|l|}{ Gender } \\
\hline Male & $6(42.9)$ \\
\hline Female & $8(57.1)$ \\
\hline \multicolumn{2}{|l|}{ Reason of application } \\
\hline GI bleeding & $4(28.6)$ \\
\hline $\begin{array}{l}\text { Gastric complaints } \\
\text { (pain, dyspepsia) }\end{array}$ & $3(21.4)$ \\
\hline Incidental & $2(14.2)$ \\
\hline Redirection & $4(21.4)$ \\
\hline Dysphagia & $1(7.1)$ \\
\hline \multicolumn{2}{|l|}{ Location } \\
\hline Lesser curvature & $6(42.9)$ \\
\hline Posterior wall & $4(28.6)$ \\
\hline Anterior wall & $3(21.4)$ \\
\hline Fundus & $1(7.1)$ \\
\hline Preoperative tumor size (cm) & $4.2(2.0-10.5)$ \\
\hline \multicolumn{2}{|l|}{ Operation type } \\
\hline Laparoscopic resection & $10(71.4)$ \\
\hline Open surgery & $4(28.6)$ \\
\hline Operative time (min) & $72.5(50-130)$ \\
\hline $\begin{array}{l}\text { Removal time of nasogastric } \\
\text { tube (d) }\end{array}$ & $1.5(1-5)$ \\
\hline First oral intake after surgery (d) & $3.5(2-28)$ \\
\hline Hospital stay $(d)$ & $6.0(5-17)$ \\
\hline \multicolumn{2}{|l|}{ Follow-up results } \\
\hline No recurrence & 13 (92.9) \\
\hline Peritoneal recurrence & $1(7.1)$ \\
\hline
\end{tabular}

Data are given as median (minimum - maximum) for continuous variables and frequency (percentage) for categorical variables. tient with schwannoma had cancer involving the submucosa, while the subserosa was involved in the other patient. Surgical margins were clean in $92.9 \%(n=13)$ of patients.

The median Ki-67 proliferation index of the patients was $3 \%$ (1-18\%). Markers found to have highest frequency of positivity were Ki-67 (11/11), C-KIT (9/12) and CD34 (8/11) (Table 2). All GISTs were C-KIT positive, and all GIST patients with results for CD34 and DOG1 were positive for these markers, while they were negative for Desmin and S100.

\section{Discussion}

Gastric tumors are common diseases with high mortality; thus, early diagnosis is important and resection with appropriate treatment options according to localization and size is the only curative approach. In this study evaluating the surgical results of benign proximal gastric tumors, the most common cause of hospital admission was gastric bleeding and complaints such as pain/dyspepsia. Tumors were most often localized in the lesser curvature of the stomach. $\mathrm{n}$ our GIST case with a tumor diameter of $5.5 \mathrm{~cm}$, which was performed with open technique, peritoneal recurrence also developed. As a result of the multidisciplinary meeting held with the oncology unit, our only case with adjacent to surgical margin was regulated

\section{Table 2. Summary of pathological results}

Tumor size (cm), median (min-max) $4.0(2.0-5.5)$

Pathological diagnosis, $\mathrm{n}(\%)$

Gastrointestinal stromal tumor $\quad 9(64.3)$

Schwannoma

$2(14.2)$

Leiomyoma

Enteric cyst

Well-differentiated

$1(7.1)$

neuroendocrine tumor

Surgical margins, $\mathrm{n}(\%)$

Clean

Focal adjacent

Ki-67 (-/+)

C-KIT (CD117) $(-/+)$

CD34 $(-/+)$

DOG1 $(-/+)$

$2 / 4$

SMA $(-/+)$

Desmin $(-/+)$

$8 / 3$

$\mathrm{S} 100(-/+)$

$9 / 3$

Mitotic rate per $50 \mathrm{HPFs}(\leq 5 />5)$ 
and followed up, and no recurrence or metastasis was detected. All GISTs were C-KIT positive.

Gastrointestinal stromal tumors can apply to the hospital with different symptoms or can be diagnosed incidentally. In our study, gastric bleeding, abdominal pain and dyspepsia were the most common presentations. Similar frequencies have been reported in other studies in the literature. Mucciarini et al. examined the clinical features of GISTs and reported that $75 \%$ of patients had gastric bleeding, 51.6\% had abdominal pain or dyspepsia, and 24.2\% had poor general condition. ${ }^{[12]}$ In another study, Scarpa et al. ${ }^{[13]}$ reviewed 46 studies examining the clinical features of GISTs, and found that GIST patients most frequently presented with gastric bleeding (5-85\%), while abdominal pain was reported as the second most common clinical presentation (4-85\%); as was the case in our study. In a previous study, Nilsson and colleagues suggested that only $69 \%$ of GIST cases diagnosed were symptomatic, and $21 \%$ of their patients had been diagnosed incidentally. ${ }^{[14]}$ In our study, $14.2 \%$ of the patients were diagnosed incidentally. Since incidental GISTs can be diagnosed in early stages, they can be treated smoothly with better surgical success and long-term results. ${ }^{[15]}$ However, due to the low number of patients in our study, the effectiveness of treatment could not be compared according to patient or diagnostic characteristics.

Laparoscopic techniques are widely used in the surgical approach to GISTs. This approach has been preferred more frequently due to its various positive features such as lower pain, faster return to daily life, and improved aesthetic appearance. ${ }^{[7]}$ In our study, it was determined that laparoscopic surgery, which has many advantages, is a successful option in gastric tumor resection. Surgical margin was clean in all patients except one. In a study examining the effectiveness of laparoscopy and resection in GIST surgery, Kim et al. ${ }^{[16]}$ found that even tumors larger than $5 \mathrm{~cm}$ could be effectively resected by laparoscopy. In a similar study, Hu et al ${ }^{[8]}$ examined the results of laparoscopic surgery and open surgery in advanced gastric cancer treatment and reported that both surgeries had similar frequency of success, complications and mortality rate. In addition, they stated that there are many advantages in choosing a laparoscopic approach in gastric surgery, and because of the importance of experience in practice, they suggested that it should be applied by experienced surgeons. ${ }^{[9,10]}$

In the surgery of benign gastric tumors, laparoscopy with intraoperative endoscopy is preferred in the surgery of small tumors, which can be seen better through the lumen. ${ }^{[16]}$ Mino and colleagues published the long-term results of GIST resection operations with the endoscopic laparoscopic technique. They reported that the operation was successfully completed without any open procedures and morbidity and mortality in any patient. The technique was suggested to be a preferable option for smaller tumors with proximal localization. ${ }^{[17]}$ In a similar study, Ojima et al. ${ }^{[18]}$ reported that laparoscopic and endoscopic technique would be an appropriate option in intraluminal lesions when compared to endoscopic intragastric surgery. Yin et al. ${ }^{[19]}$ examined 3 different minimally invasive surgical techniques in GIST operations. They emphasized that the decision of surgical approach should be made according to the location and size of the tumor, and that endoscopic support may be important, especially in intraluminal cases. In our study, 7 of the 10 laparoscopic operations were accompanied by endoscopy. During the procedure, resection procedures and control of passage and stapler line were performed by identifying tumor size and exact localization more clearly with endoscopy. Although the number of patients evaluated in the current study is low, it can be said that the results of patients undergoing laparoscopic endoscopy are also very successful. In addition to its advantageous results for patients, it can be said that this technique is a preferable option for surgeons.

Gastrointestinal stromal tumors are most commonly a lesion of the stomach. Localization and size of the tumor in the stomach are important variables that affect the surgeon's decision in terms of laparoscopy. In our study, almost half of the tumors were localized in the lesser curvature of the stomach. In studies evaluating the results of laparoscopy in GIST surgery, it is seen that tumor localization shows varying distributions between studies. This may be related to the decisions of surgeons with regard to surgical approach, the characteristics of the patients in question, and the availability of other surgical approaches. For example, Xiong et al. ${ }^{[20]}$ published laparoscopic resection results of proximal GISTs and reported that the tumor was located in the fundus and greater curvature in more than half of the cases. Stanek et al., ${ }^{[21]}$ in a similar study, reported that half of the tumors were located in the large curvature. Many other studies have reported different localization frequencies. When determining the surgical technique, the localization of the tumor and the experience of the surgeon are very important. However, there are also studies in the literature reporting that the results of open surgery or laparoscopic 
approach are similar in the resection of tumors localized at unfavorable regions. Huang and colleagues evaluated the results of laparoscopic surgery of GISTs localized in unfavorable sites. They reported that 5-year survival and disease-free survival were similar between laparoscopic surgery and open surgery, but the acute period results of patients undergoing laparoscopy were more successful. They also reported that the frequency of complications was lower after the resection of GISTs in unfavorable sites with laparoscopic surgery. ${ }^{[2]}$ Therefore, the option of laparoscopy should be taken into account in the resection of localized tumors in unfavorable sites, depending on the experience of the surgeon performing the operation.

Tumors can be diagnosed based on the detection of different markers, or their treatment can be determined based on these results. In our study, many tumor markers were examined, and more than half of the patients were found to have positive results for Ki-67 (11/11), C-KIT (9/12) and CD34 (8/11). All C-KIT, CD34 and DOG1 positive patients were diagnosed with GIST, while all of them were also negative for desmin and S100. KIT is a tyrosine kinase receptor, which is secreted by many interstitial cells of Cajal. Since GISTs are derived from these cells, almost all of them are KIT positive. ${ }^{[23,24]}$ These cells also contain DOG1 protein. However, since DOG1 can be released from different cells, GIST specificity is usually not very high. ${ }^{[25,26]}$ On the other hand, CD34 is a surface glycoprotein responsible for adhesion and is often positive in gastric-located GISTs. ${ }^{[24]}$ Desmin and S100 are usually markers for GIST exclusion and can rarely be positive in GISTs. ${ }^{[27]}$ Therefore, it is apparent that our observations with these markers were compatible with the literature on this topic.

Proximal tumors of the stomach, especially proximal GISTs, are extremely rare; thus, limiting the number of patients that could be included in the study. In conjunction with this situation, features such as complication rates and morbidity/mortality could not be calculated. Furthermore, we did not assess the possible effects of various other variables (surgical technique, various tumor markers, time before and after surgery, age of patients, tumor stage, additional diseases, surgeon's experience, etc.) on patients and diagnoses. Finally, all the limitations that can be encountered in an observational study are also valid for our study. Such situations may have caused misinterpretation of the results.

In conclusion, resection of benign proximal gastric tumors with laparoscopic endoscopic or open surgery provides successful results. There was seen no difference between open surgical, laparoscopic and endoscopic techniques in the long-term follow-ups. In this study, major morbidity or mortality did not develop in the postoperative period. In the resection of benign proximal gastric tumors, according to the exact localization of the tumor and the experience of the surgeon, laparoscopic endoscopy should be considered as a primary option due to its known advantages and ease of exploration.

\section{Disclosures}

Peer-review: Externally peer-reviewed.

Conflict of Interest: None declared.

Authorship Contributions: Concept - E.H., M.S.E., M.E.; Design - E.H., M.S.E., M.E.; Supervision - M.S.E.; Materials - E.H.; Data collection and/or processing - E.H.; Analysis and/or interpretation - E.H.; Literature search - E.H.; Writing - E.H., M.S.E., M.E.; Critical review - E.H., M.S.E., M.E.

\section{References}

1. Bray F, Ferlay J, Soerjomataram I, Siegel RL, Torre LA, Jemal A. Global cancer statistics 2018: GLOBOCAN estimates of incidence and mortality worldwide for 36 cancers in 185 countries. CA Cancer J Clin 2018;68(6):394-424. [CrossRef]

2. Sugano K. Screening of gastric cancer in Asia. Best Pract Res Clin Gastroenterol 2015;29:895-905. [CrossRef]

3. Sitarz R, Skierucha M, Mielko J, Offerhaus GJA, Maciejewski R, Polkowski WP. Gastric cancer: epidemiology, prevention, classification, and treatment. Cancer Manag Res 2018;10:239-48. [CrossRef]

4. Rawla P, Barsouk A. Epidemiology of gastric cancer: global trends, risk factors and prevention. Prz Gastroenterol 2019;14:26-38. [CrossRef]

5. Mukaisho K, Nakayama T, Hagiwara T, Hattori T, Sugihara H. Two distinct etiologies of gastric cardia adenocarcinoma: interactions among $\mathrm{pH}$, Helicobacter pylori, and bile acids. Front Microbiol 2015;6:412. [CrossRef]

6. Orditura M, Galizia G, Sforza V, Gambardella V, Fabozzi A, Laterza MM, et al. Treatment of gastric cancer. World J Gastroenterol 2014;20:1635-49. [CrossRef]

7. Chen K, Zhou YC, Mou YP, Xu XW, Jin WW, Ajoodhea H. Systematic review and meta-analysis of safety and efficacy of laparoscopic resection for gastrointestinal stromal tumors of the stomach. Surg Endosc 2015;29:355-67. [CrossRef]

8. Hu Y, Huang C, Sun Y, Su X, Cao H, Hu J, et al. Morbidity and Mortality of Laparoscopic Versus Open D2 Distal Gastrectomy for Advanced Gastric Cancer: A Randomized Controlled Trial. J Clin Oncol 2016;34:1350-7. [CrossRef]

9. Liang JW, Zheng ZC, Zhang JJ, Zhang T, Zhao Y, Yang W, et al. Laparoscopic versus open gastric resections for gastric 
gastrointestinal stromal tumors: a meta-analysis. Surg Laparosc Endosc Percutan Tech 2013;23:378-87. [CrossRef]

10. Koh YX, Chok AY, Zheng HL, Tan CS, Chow PK, Wong WK, et al. A systematic review and meta-analysis comparing laparoscopic versus open gastric resections for gastrointestinal stromal tumors of the stomach. Ann Surg Oncol 2013;20:3549-60. [CrossRef]

11. Siewert JR, Hölscher AH, Becker K, Gössner W. Cardia cancer: attempt at a therapeutically relevant classification. [Article in German] Chirurg 1987;58:25-32.

12. Mucciarini C, Rossi G, Bertolini F, Valli R, Cirilli C, Rashid I, et al. Incidence and clinicopathologic features of gastrointestinal stromal tumors. A population-based study. BMC Cancer 2007;7:230. [CrossRef]

13. Scarpa M, Bertin M, Ruffolo C, Polese L, D'Amico DF, Angriman I. A systematic review on the clinical diagnosis of gastrointestinal stromal tumors. J Surg Oncol 2008;98:384-92.

14. Nilsson $B$, Bümming $P$, Meis-Kindblom JM, Odén A, Dortok A, Gustavsson B, et al. Gastrointestinal stromal tumors: the incidence, prevalence, clinical course, and prognostication in the preimatinib mesylate era--a population-based study in western Sweden. Cancer 2005;103:821-9. [CrossRef]

15. Chan $\mathrm{CH}$, Cools-Lartigue J, Marcus VA, Feldman LS, Ferri LE. The impact of incidental gastrointestinal stromal tumours on patients undergoing resection of upper gastrointestinal neoplasms. Can J Surg 2012;55:366-70. [CrossRef]

16. Kim JJ, Lim JY, Nguyen SQ. Laparoscopic resection of gastrointestinal stromal tumors: Does laparoscopic surgery provide an adequate oncologic resection?. World J Gastrointest Endosc 2017;9:448-55. [CrossRef]

17. Mino JS, Guerron AD, Monteiro R, El-Hayek K, Ponsky JL, Patil DT, et al. Long-term outcomes of combined endoscopic/laparoscopic intragastric enucleation of presumed gastric stromal tumors. Surg Endosc 2016;30:1747-53. [CrossRef]

18. Ojima T, Nakamura $M$, Nakamori $M$, Takifuji $K$, Hayata $K$, Katsuda $M$, et al. Laparoscopic and endoscopic cooperative surgery is a feasible treatment procedure for intraluminal gastric gastrointestinal stromal tumors compared to endoscopic intragastric surgery. Surg Endosc 2018;32:351-7.

19. Yin X, Yin Y, Chen H, Shen C, Tang S, Cai Z, et al. Comparison Analysis of Three Different Types of Minimally Invasive Procedures for Gastrointestinal Stromal Tumors $\leq 5 \mathrm{~cm}$. J Laparoendosc Adv Surg Tech A 2018;28:58-64. [CrossRef]

20. Yin X, Yin Y, Chen H, Shen C, Tang S, Cai Z, et al. Comparison Analysis of Three Different Types of Minimally Invasive Procedures for Gastrointestinal Stromal Tumors $\leq 5 \mathrm{~cm}$. J Laparoendosc Adv Surg Tech A 2018;28:58-64. [CrossRef]

21. Stanek $M$, Pisarska $M$, Budzyńska D, Rzepa A, Pędziwiatr $M$, Major $P$, et al. Gastric gastrointestinal stromal tumors: clinical features and short- and long-term outcomes of laparoscopic resection. Wideochir Inne Tech Maloinwazyjne 2019;14:176-81. [CrossRef]

22. Huang CM, Chen QF, Lin JX, Lin M, Zheng $\mathrm{CH}$, Li P, et al. Can laparoscopic surgery be applied in gastric gastrointestinal stromal tumors located in unfavorable sites?: A study based on the NCCN guidelines. Medicine (Baltimore) 2017;96:e6535. [CrossRef]

23. Hirota S, Isozaki K, Moriyama Y, Hashimoto K, Nishida T, Ishiguro $S$, et al. Gain-of-function mutations of c-kit in human gastrointestinal stromal tumors. Science 1998;279:577-80.

24. Hirota S. Differential diagnosis of gastrointestinal stromal tumor by histopathology and immunohistochemistry. Transl Gastroenterol Hepatol 2018;3:27. [CrossRef]

25. West RB, Corless CL, Chen X, Rubin BP, Subramanian S, Montgomery $\mathrm{K}$, et al. The novel marker, DOG1, is expressed ubiquitously in gastrointestinal stromal tumors irrespective of KIT or PDGFRA mutation status. Am J Pathol 2004;165:107-13.

26. Swalchick W, Shamekh R, Bui MM. Is DOG 1 Immunoreactivity Specific to Gastrointestinal Stromal Tumor?. Cancer Control 2015;22:498-504. [CrossRef]

27. Connolly EM, Gaffney E, Reynolds JV. Gastrointestinal stromal tumours. Br J Surg 2003;90:1178-86. [CrossRef] 